\title{
The physical chemistry of the enigmatic phospholipid diacylglycerol pyrophosphate
}

\author{
Liza Strawn' , Amy Babb' ${ }^{2}$, Christa Testerink ${ }^{3}$ and Edgar Eduard Kooijman ${ }^{4}$ \\ ${ }^{1}$ Biotechnology Program, Kent State University, Kent, OH, USA \\ 2 Department of Chemistry, Kent State University, Kent, OH, USA \\ ${ }^{3}$ Section of Plant Physiology, Swammerdam Institute for Life Sciences, University of Amsterdam, Amsterdam, Netherlands \\ ${ }^{4}$ Department of Biological Sciences, Kent State University, Kent, OH, USA
}

Edited by:

Xuemin Wang, University of

Missouri-St. Louis, USA

Reviewed by:

Robert V. Stahelin, Indiana University School of Medicine-South Bend, USA Alain Zachowski, Université Pierre et Marie Curie, France

\section{*Correspondence:}

Edgar Eduard Kooijman, Department of Biological Sciences, Kent State University, PO Box 5190, Kent, $\mathrm{OH}$ 44242, USA.

e-mail: ekooijma@kent.edu
Phosphatidic acid (PA) is a lipid second messenger that is formed transiently in plants in response to different stress conditions, and plays a role in recruiting protein targets, ultimately enabling an adequate response. Intriguingly, this increase in PA concentration in plants is generally followed by an increase in the phospholipid diacylglycerolpyrophosphate (DGPP), via turnover of PA. Although DGPP has been shown to induce stress-related responses in plants, it is unclear to date what its molecular function is and how it exerts its effect. Here, we describe the physicochemical properties, i.e., effective molecular shape and charge, of DGPP. We find that unlike PA, which imparts a negative curvature stress to a (phospho)lipid bilayer, DGPP stabilizes the bilayer phase of phosphatidylethanolamine $(P E)$, similar to the effect of phosphatidylcholine (PC). DGPP thus has zero curvature. The $\mathrm{pKa}_{2}$ of the phosphomonoester of DGPP is $7.44 \pm 0.02$ in a PC bilayer, compared to a $\mathrm{pKa}_{2}$ of 7.9 for PA. Replacement of half of the PC with PE decreases the $\mathrm{pKa}_{2}$ of DGPP to $6.71 \pm 0.02$, similar to the behavior previously described for PA and summarized in the electrostatic-hydrogen bond switch model. Implications for the potential function of DGPP in biomembranes are discussed.

Keywords: phosphatidic acid, phospholipid signaling, electrostatic hydrogen bond switch, lipid second messenger, effective lipid shape, ionization, dgpp, diacylglycerol pyrophosphate

\section{INTRODUCTION}

Biological membranes are crucial for the function of all cells in all living organisms. They act as selective barriers, within, or around a cell. Biomembrane structure and function is not only critically influenced by membrane proteins, but also by their diverse lipid composition. The phase behavior and structure of many membrane lipids has been determined in great detail (e.g., see Gennis, 1989; Marsh, 2012). Despite this wealth of information, much still needs to be learned in order to fully appreciate the defining properties of membrane lipids and understand the huge diversity of lipids found in biological membranes. In particular, lipids that function as important signaling molecules and often occur in membranes in minute and rapidly changing concentrations deserve further attention.

Phosphatidic acid (PA) has emerged as a lipid-signaling molecule in all eukaryotes. In plants, it plays a role in stress signaling. It is rapidly generated in response to salinity, cold, wounding, and pathogen attack. Recent data from plants, mammals, and yeast indicate that the formation of PA functions as a membranelocalized signal, affecting downstream responses by binding specific protein targets. These include protein kinases and phosphatases, and various proteins involved in vesicular trafficking (Stace and Ktistakis, 2006; Testerink and Munnik, 2011). Moreover, because of its small polar headgroup, adding negative charge and curvature to the bilayer, PA formation has profound effects on membrane architecture, and could reduce the energy barrier for vesicle fission and fusion, even without interaction with proteins (Roth, 2008).

Stress induced increases in plants are always transient, and several enzymes have been identified that function in attenuation of the PA signal (Testerink and Munnik, 2011). One of these routes is phosphorylation of PA to diacylglycerol pyrophosphate (DGPP) by PA kinase [(PAK), see Figure 5 for structure of DGPP and PA] This pathway was first discovered in vivo through ${ }^{32} \mathrm{P}_{\mathrm{i}}$-labeling studies in the green alga Chlamydomonas, where treatment with the G-protein activator mastoparan resulted in a dramatic increase in PA, which was accompanied by an increase in another, unknown lipid, which later turned out to be DGPP (Munnik et al., 1995, 1996).

Although present in minute amounts under control conditions, DGPP levels are induced upon several biotic and abiotic cues. DGPP levels increase upon hyperosmotic stress treatment of Chlamydomonas, the resurrection plant Craterostigma plantagineum, rice leaves and cell suspension cultures of tomato, alfalfa, and Arabidopsis thaliana (Pical et al., 1999; Munnik et al., 2000; Darwish et al., 2009). Interestingly, DGPP is also formed upon elicitation by both pathogenic and beneficial microorganisms. Treatment of suspension-cultured tomato cells with pathogen effectors (van der Luit et al., 2000; de Jong et al., 2004) and Nod factor treatment of Vicia sativa seedlings and Medicago cell suspensions (den Hartog et al., 2001, 2003) all result in DGPP increases. Moreover, treatment of cells or seeds with the plant stress hormone ABA was 
also shown to induce an increase in DGPP (Katagiri et al., 2005; Zalejski et al., 2005). Interestingly, downstream ABA responses, such as plasma membrane anion currents and Rab18 gene expression, could be induced by treatment of the cells with C18:1 DGPP, indicating a role for DGPP in ABA signaling (Zalejski et al., 2005, 2006).

The kinetics of formation of PA and DGPP, as well as labeling techniques, have shown that DGPP is synthesized by phosphorylation of PA (Munnik et al., 1996). However, the gene encoding the PAK activity still has not been identified to date, severely hampering any genetic analysis of the pathway. In turn, DGPP can be dephosphorylated again to PA by an enzyme called DGPP phosphatase (DPP). Four genes encoding DPPs have been identified in Arabidopsis thaliana, called AtLPP1-4 (Jeannette et al., 2010). Interestingly, an Atlpp2 insertion mutant exhibits increased levels of both DGPP and PA during germination (Katagiri et al., 2005). This suggests that the mutant is disturbed in both DPP and PAP activity, consistent with AtLPP2's similar affinity for both substrates (Pierrugues et al., 2001). The lpp2 mutant was shown to be more sensitive to ABA than wild-type with regard to seed germination (Katagiri et al., 2005) and ABA-induced guard cell responses in the leaf (Paradis et al., 2011).

\section{A FUNCTIONAL ROLE OF DGPP IN PLANTS: DEFINING MOLECULAR PROPERTIES}

Fifteen years after its discovery in plants, our understanding of DGPP formation and degradation in plants is still fragmentary and its molecular function is still completely unknown. A thorough in vitro investigation of molecular properties such as ionization and membrane packing properties is of key importance to understand how DGPP affects both membrane architecture and protein binding. Recent physicochemical studies of PA have led to the electrostatic-hydrogen bond switch model, and turned out to be crucial to explain some of its physiological functions (e.g., binding of Opil to PA). Young et al. elegantly showed how the ionization properties of PA explain the binding of the transcription repressor Opil and suggested that PA acts as a $\mathrm{pH}$ sensor in yeast linking metabolism and lipid synthesis (Ktistakis, 2010; Young et al., 2010; Orij et al., 2011; Shin and Loewen, 2011). In analogy to PA, the physicochemical properties of DGPP are likely crucial for a full understanding of the physiology of this unusual plant lipid. Aside from one recent study describing the surface properties of DGPP in Langmuir monolayers (Villasuso et al., 2010), there is, to the best of our knowledge, no physicochemical data available for this lipid.

Here, we describe our initial characterization of the effective lipid molecular shape and the ionization properties of DGPP in simple model membrane systems. These data will set a crucial baseline against which to compare data from more complicated lipid and buffer compositions. We show that ionization properties of the phosphomonoester of DGPP mimic those of PA, i.e., follow the electrostatic-hydrogen bond switch model. These data thus suggest that DGPP is able to interact with cationic protein domains and may possess its own unique signaling functions. Contrary to PA though, DGPP is not a cone shaped lipid, i.e., is not able to impart a negative curvature stress to the membrane and facilitate the insertion of hydrophobic protein domains into the membrane.
Thus, DGPP packing properties more closely resemble those of PC, completely opposite to PA. This finding is likely to have significant implications for proteins binding to DGPP. Additionally, the presence of two phosphates could significantly change the presentation (orientation and position) of the phosphomonoester of DGPP in the lipid headgroup-acyl chain interface.

\section{RESULTS}

\section{DGPP STABILIZES THE BILAYER PHASE}

A qualitative description of effective molecular shape for membrane lipids is conveniently achieved by determining their effect on the bilayer to hexagonal $\left(\mathrm{L}_{\alpha}-\mathrm{H}_{\mathrm{II}}\right)$ phase transition temperature of dielaidoylphosphatidylethanolamine (DEPE; Rand et al., 1990; Kooijman et al., 2003). Dielaidoylphosphatidylethanolamine is chosen for its convenient $\mathrm{L}_{\alpha}$ to $\mathrm{H}_{\text {II }}$ phase transition temperature of $65^{\circ} \mathrm{C}$ and matching number of acyl chain carbons, and unsaturation. An additional benefit of DEPE over other PE's is that many previous studies used this lipid as matrix and can thus be used to compare our current results (e.g., Rand et al., 1990; Fuller and Rand, 2001; Szule et al., 2002; Kooijman et al., 2003).

Here we used differential scanning calorimetry (DSC) to determine the effect of $5 \mathrm{~mol} \%$ DGPP on the $\mathrm{H}_{\text {II }}$ phase transition temperature of DEPE, and compare those data with that of other well-known membrane lipids. Figure 1A shows representative DSC scans for the $\mathrm{L}_{\alpha}-\mathrm{H}_{\text {II }}$ phase transition of DEPE, and DEPE containing $5 \mathrm{~mol} \%$ of DOPE, DOPC, DGPP, DOPA, and LPC as indicated. In order to show the consistency of these results and to quantify the shift in $\mathrm{T}_{\mathrm{H}}$ we plotted the $\mathrm{T}_{\mathrm{H}}$ for each of these conditions in Figure 1B. Shown is the average of three independent experiments and samples. The error bar denotes the calculated SD. From these data it is clear that unlike DOPA, DGPP increases the $\mathrm{L}_{\alpha}$ to $\mathrm{H}_{\mathrm{II}}$ phase transition temperature of DEPE and the data is highly reproducible. In the case of the DOPA-DEPE and DGPPDEPE mixtures the data shows two peaks (see Figure 1A). This likely represents a demixing of the mixtures at the $\mathrm{L}_{\alpha}-\mathrm{H}_{\text {II }}$ phase transition, resulting in, respectively, a DOPA and DGPP rich and poor phase. We will comment on this in the discussion. Interestingly, when we compare the effect induced by DGPP with that of DOPC (also shows a small amount of peak splitting and thus likely a DOPC poor and rich phase at the transition) we notice that both are essentially equal. This suggests that DGPP has a cylindrical shape and will thus stabilize the bilayer phase of DEPE. Consistent with our previous results for DOPA we again show that DOPA has essentially the same effect on the phase transition temperature of DEPE as DOPE (Kooijman et al., 2003). Hence DOPA and DOPE, under experimental conditions of $150 \mathrm{mM} \mathrm{NaCl}$ and at $\mathrm{pH} 7.2$ $\left(\mathrm{pH}\right.$ at $\left.22^{\circ} \mathrm{C}\right)$, have a similar effective molecular shape. We showed that the same is true for a quantitative measure of effective molecular shape, i.e., the spontaneous curvature (Kooijman et al., 2005a). Not surprisingly, LPC strongly stabilizes the bilayer phase of DEPE again consistent with our previous work (Kooijman et al., 2003).

\section{IONIZATION OF DGPP IN DOPC}

Effective lipid molecular shape is sensitive to environmental factors such as temperature, ionic strength, and $\mathrm{pH}$. In the case of DGPP with a pyrophosphate headgroup, these parameters will influence the charge carried by this group. Additionally headgroup charge 


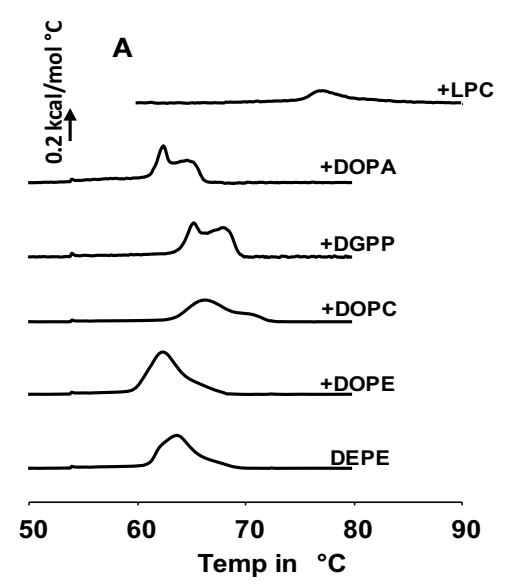

FIGURE 1 | (A) differential scanning calorimetry data for $5 \mathrm{~mol} \%$ of indicated phospholipid in DEPE. Bottom curve is DEPE control. Shown is the third upscan of each experiment. (B) Means of the $T_{\text {HII }}$ from three

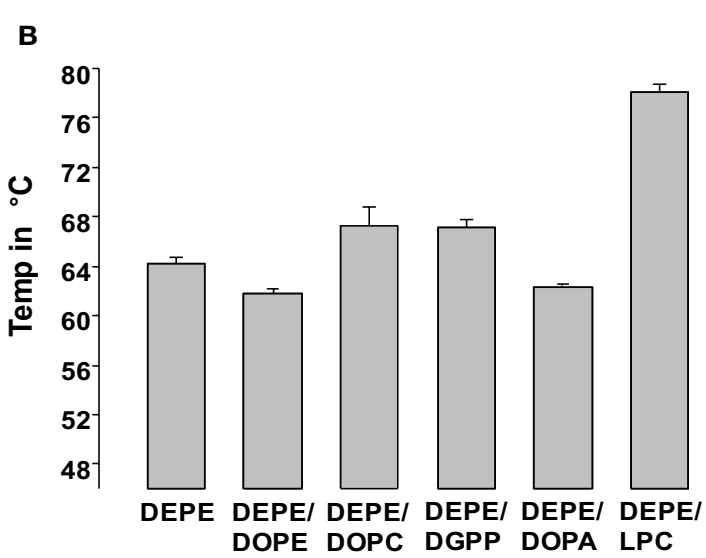

independent experiments. In the case of DOPA, DGPP, and DOPC only the $T_{H I I}$ value for the furthest and most prominent peak (up or down from DEPE) is shown. and how this charge depends on $\mathrm{pH}$ and membrane lipid composition is of critical importance to understand binding of cationic protein domains and lipid-lipid interactions. Hence we set out to determine the negative charge (pKa's) of the phosphomonoester of DGPP.

Ionization of small concentrations of anionic lipids in a multilamellar matrix (multilamellar vesicles, MLVs) of zwitterionic lipids is most conveniently achieved using solid state (magic angle spinning, MAS) NMR techniques. Static ${ }^{31} \mathrm{P}$ NMR of MLVs results in a large chemical shift anisotropy (CSA) and does not allow for discrimination of the individual chemical shift of the phospholipids making up the phospholipid bilayer (Hauser, 1989; Swairjo et al., 1994). In the $\mathrm{L}_{\alpha}$ phase the spectra are characterized by a low field shoulder and high field peak as indicated in Figure 2. These spectra are representative of large MLVs. The static spectra shown in Figure 2 thus confirm that the lipid dispersions used in our studies form lipid bilayers. We thus used ${ }^{31} \mathrm{P}$ MAS NMR to prepare $\mathrm{pH}$ titration curves for the phosphomonoester of DGPP in DOPC alone and in an equimolar mixture of DOPC and DOPE. Figure 3A shows the ${ }^{31} \mathrm{P}$ NMR spectra for $5 \mathrm{~mol} \%$ DGPP in DOPC plotted as a function of $\mathrm{pH}$. The PC peak is clearly visible as the low field peak near 0 ppm, and the two DGPP peaks are also clearly visible upfield from PC due to the additional shielding by the electrons of the pyrophosphate group. The peak furthest upfield is identified as the phosphodiester peak of DGPP as it is shielded the most, as expected. It is broadened over the entire $\mathrm{pH}$ range studied. The phosphomonoester peak is identified as the downfield peak from the phosphodiester peak and clearly shifts as a function of $\mathrm{pH}$ just as we observed previously for other lipid phosphomonoesters (Kooijman et al., 2005b, 2007, $2008,2009)$. The phosphomonoester peak of DGPP is seen to split into two peaks in the regions of the titration curve where charge is constant and indicates the spin-spin coupling of the two phosphate groups in the pyrophosphate (Friebolin, 2011). In regions where charge changes as a function of $\mathrm{pH}$ the peak is somewhat broadened and the $\sim 15 \mathrm{~Hz}$ splitting is no longer observable.

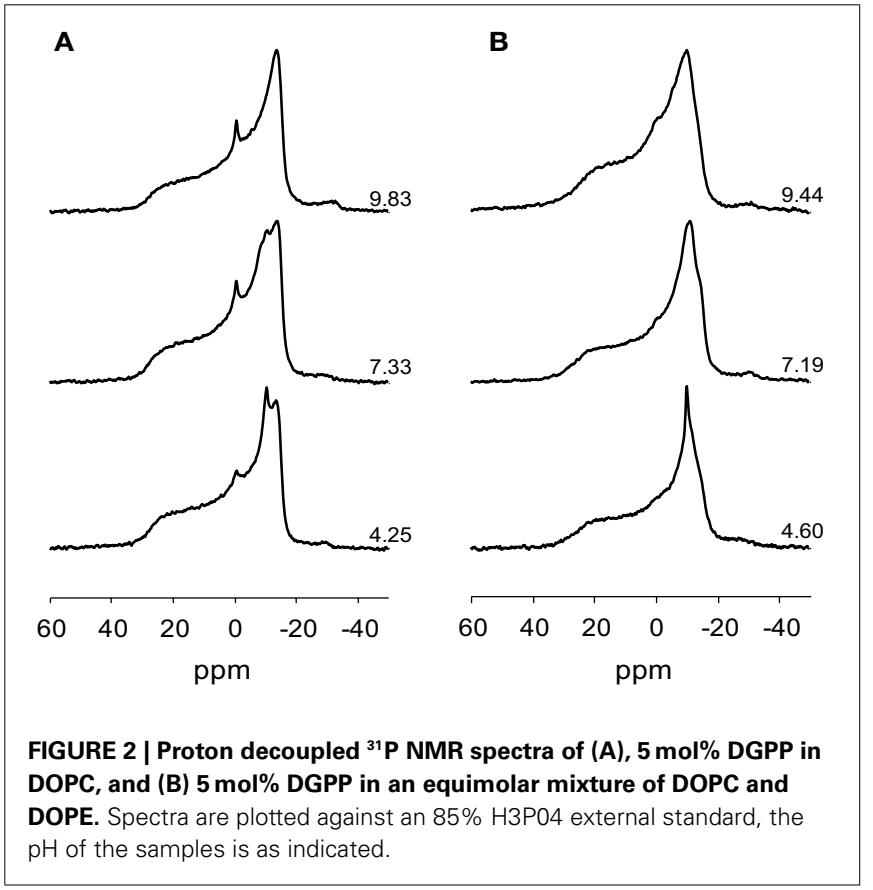

In order to determine the ionization constants for the phosphomonoester group we determined the peak positions using Bruker Topspin software and plotted these as a function of $\mathrm{pH}$ to create the titration curve for DGPP in DOPC shown in Figure 3C (red circles). When the peak is split in two due to spin-spin coupling we take the average of the two peak positions as this indicates the true chemical shift of the phosphate (Friebolin, 2011). The solid line(s) in Figure 3C are non-linear least squares fits using Eq. 1. The pKa values for DGPP in DOPC are summarized in Table 1.

Phosphatidylcholine, while a major zwitterionic lipid, is usually not the only one present and membranes generally contain a significant amount of PE. Hence we also determined the ionization behavior of DGPP in an equimolar mixture of DOPC and DOPE. 

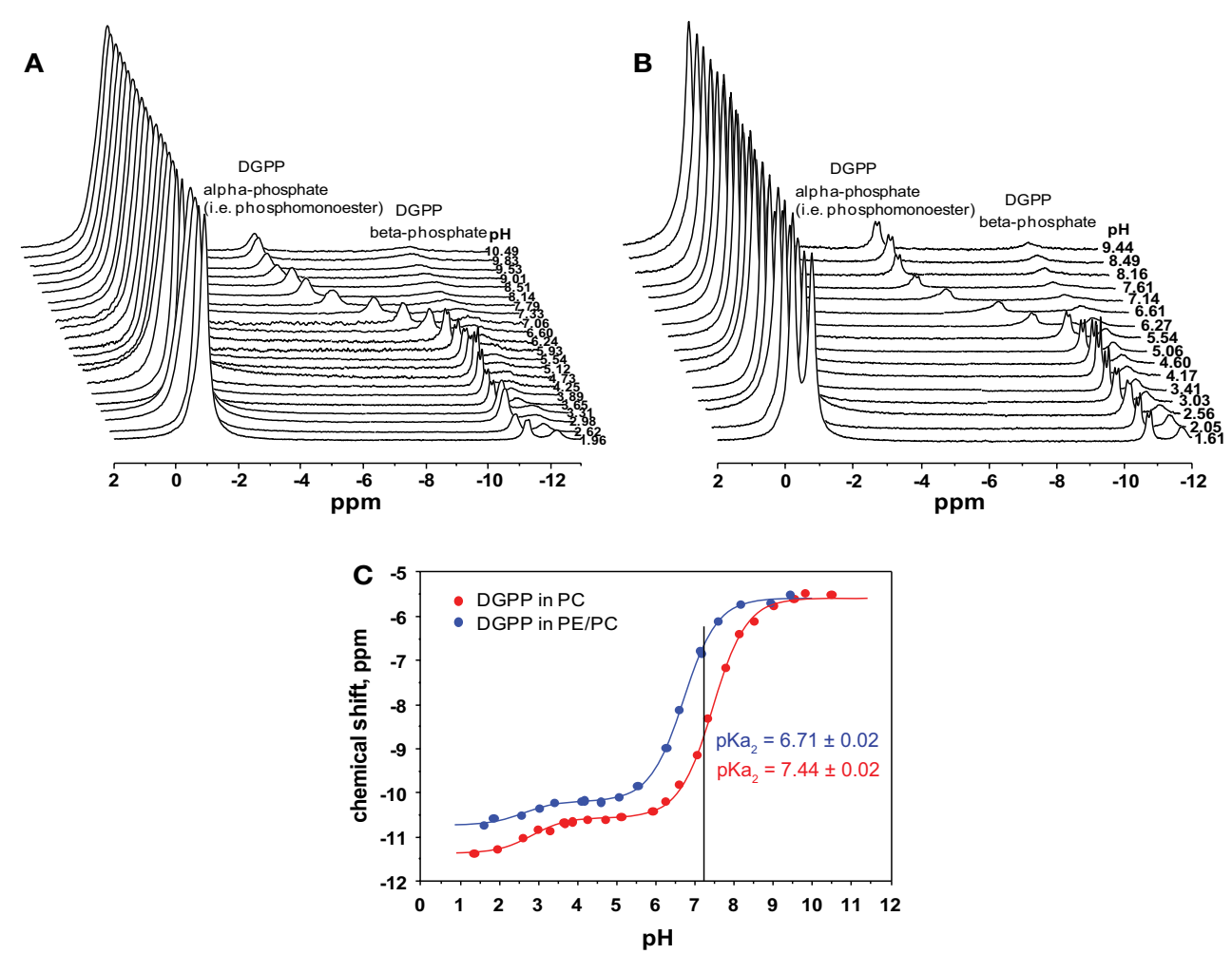

FIGURE 3 | ${ }^{31} \mathrm{P}$ NMR spectra for $5 \mathrm{~mol} \%$ dioleoyl diacylglycerol pyrophosphate (18:1 DGPP) in (A), DOPC, and (B), an equimolar mixture of DOPC and DOPE as a function of pH. (C), $\mathrm{pH}$ titration curves for $5 \mathrm{~mol} \%$
DGPP in DOPC (red curve) and 5 mol\% DGPP in DOPC-DOPE 1:1 (blue curve). Lines are best non-linear least squares fits to a Henderson-Hasselbalch type equation.
Table 1 | lonization constants for 5 mol\% DGPP in multilamellar vesicles of DOPC and DOPC/DOPE; and for $10 \mathrm{~mol} \% \mathrm{PA}$ in multilamellar vesicles of DOPC.

\begin{tabular}{lll}
\hline & $\mathbf{p K a}_{\mathbf{1}}$ & $\mathbf{p K \mathbf { a } _ { \mathbf { 2 } }}$ \\
\hline DGPP in DOPC & $2.82 \pm 0.15$ & $7.44 \pm 0.02$ \\
DGPP in DOPC/DOPE $(1: 1)$ & $2.61 \pm 0.22$ & $6.71 \pm 0.02$ \\
PA in DOPC & $3.2 \pm 0.3$ & $7.92 \pm 0.03$
\end{tabular}

${ }^{2}$ Data taken from Kooijman et al. (2005b).

The ${ }^{31} \mathrm{P}$ NMR spectra as a function of $\mathrm{pH}$ are shown in Figure 2B. For the two low field peaks, the PE peak is found slightly down field of that for PC (indicating the deshielding of the phosphate in $\mathrm{PE}$ compared to PC). Interestingly, the phosphomonoester peak splitting is considerably more pronounced in the PE containing membrane compared to the membrane containing just PC (compare Figures 3A,B). Again we plot the peak position of the phosphomonoester peak of DGPP as a function of $\mathrm{pH}$ and the data is shown in Figure 3C (blue circles), and the pKa values are summarized in Table 1. It should be noted that at high $\mathrm{pH}$ the amine of PE deprotonates resulting in a negatively charged lipid. This alters membrane electrostatics and might affect the charge of DGPP. However we do not observe a large effect as the data still fit a simple Henderson-Hasselbalch behavior $\left(R^{2}>0.99\right)$. This is likely due to the fact that at high pH DGPP (phosphomonoester) is already fully deprotonated and a resulting increase in the negative electrostatic potential does not additionally affect the charge of DGPP's phosphomonoester.

Interestingly the titration curves for DGPP in the DOPC and the DOPC-DOPE 1:1 model membrane do not overlap. In fact the degree of ionization of the phosphomonoester of DGPP in the PE containing membrane is considerably larger compared to the ionization of DGPP in the PC membrane as indicated by the shift of the titration curve to lower $\mathrm{pH}$ values. This striking difference in degree of ionization (and thus $\mathrm{pKa}$ ) is also indicated by the straight line drawn at $\mathrm{pH}$ 7.2. In the case of the PC:DGPP membrane the line hits the titration curve significantly lower compared to the PC:PE:DGPP curve. The higher on the curve the higher the total negative charge, keeping in mind that this position indicates the percentage of phosphomonoester groups that carry -1 and -2 negative charges (fractional charges being non-physical). These data are consistent with the electrostatic-hydrogen bond switch model that we described previously for PA (Kooijman et al., 2007; Kooijman and Testerink, 2010).

\section{DISCUSSION}

Taken together these data present an intriguing picture of the physicochemical properties of DGPP. On the one hand the effective molecular shape of DGPP is opposite to that of PA, but on the other the ionization properties of the phosphomonoester group of DGPP follows the same behavior previously described for PA. 
Here, we discuss the potential implications of these data for the function of DGPP in biological membranes.

\section{EFFECTIVE LIPID SHAPE OF DGPP}

We compared the effect of a small concentration of DGPP on the $\mathrm{L}_{\alpha}-\mathrm{H}_{\text {II }}$ phase transition temperature of DEPE to that of wellknown lipids such as DOPE, DOPA, DOPC, and LPC. The packing properties of these model phospholipids can be divided in the following three groups: negative curvature (DOPE and DOPA), zero curvature (DOPC), and positive curvature (LPC). Our data clearly show that DGPP behaves in a manner consistent with the behavior of DOPC, and thus indicate that at physiological $\mathrm{pH}$ and salt concentration DGPP can be considered a membrane stabilizing lipid, contributing negligible curvature to a membrane.

The zero curvature (cylindrical effective molecular shape) of DGPP is caused by several factors. The main factor is the increased steric interaction in the headgroup region caused by a larger headgroup. This headgroup is not only larger due to the pyrophosphate vs. phosphomonoester of PA, but also due to additional water molecules that hydrate this group. It is unlikely that the observed bilayer stabilization by DGPP in our experiments is caused only by an expansion of the bilayer due to charge repulsion. Although DGPP carries more negative charge than PA, in fact highly charged lipids have been found to exhibit favorable interactions that rather result in a condensing effect. Janmey and coworkers for example suggest that favorable interactions between highly charged polyphosphoinositides are potentially mediated by hydrogen bond interactions (Levental et al., 2008a,b). We recently showed, albeit indirectly, that $\mathrm{PI} 4,5 \mathrm{P}_{2}$ likely forms domains in a PC bilayer, using similar ${ }^{31} \mathrm{P}$ NMR titration curves as employed here for DGPP (Kooijman et al., 2009). Also, Villasuso et al. (2010) recently showed a condensing effect of DGPP monolayers in the presence of PA. This latter finding suggests interesting possibilities to the potential role of DGPP in biological membranes. In conclusion it should be noted that despite significant negative charge PA does form the hexagonal phase with PE, and in fact destabilizes the PE lipid bilayer. Charge effects certainly play a role in the effective molecular shape of DGPP but are not, a priori, the dominant factor.

The cylindrical nature of DGPP is in stark contrast to our previous data for PA which destabilizes the bilayer under identical physiological conditions. Our data would thus favor a model for the function of DGPP which is opposite that of PA. Along the same line these data indicate that if PA is converted to DGPP upon plant cell exposure to stress, the resulting DGPP might no longer favor the binding of PA specific proteins that require the negative curvature stress induced by PA to effectively bind to the membrane. Additionally, conversion of PA to DGPP in plant cell membranes undergoing membrane fission or fusion might result in an inhibition of membrane fusion (fission).

\section{DEMIXING OF DGPP AND PA AT THE $\mathrm{L}_{\alpha}-\mathrm{H}_{\boldsymbol{I}}$ PHASE TRANSITION}

We very consistently observed that the phase transition for DEPE witnessed by DSC indicates some degree of demixing of DEPE and DGPP (and DOPA). This observation might be explained by the fact that PE is likely to form hydrogen bonds with the pyrophosphate headgroup of DGPP. The effect of PE on the ionization behavior of the phosphomonoester group of DGPP is a sign of this hydrogen bonding (discussed below). Alternatively it might be caused by a combination of non-ideal mixing of the acyl chains (trans double bonds for DEPE and cis double bonds for DOPA and DGPP) combined with more favorable interactions (through hydrogen bonds) between the headgroups. It is likely not caused by complete phase separation as we took care in preparing the lipid films at a temperature above the $T_{\mathrm{m}}$ for DEPE. Whatever the reason for this intriguing observation our data demonstrate that DGPP and DOPA have an opposite effect on the phase transition; DGPP stabilizes the bilayer and PA destabilizes the bilayer of DEPE. In future work we plan to follow up on this observation and investigate whether or not PE or other hydrogen bond donors are able to phase separate with DGPP from the rest of the membrane in the physiologically relevant $\mathrm{L}_{\alpha}$ phase. Non-ideal mixing and fluidfluid immiscibility was studied for PE-PA mixtures previously and depending on the acyl chain composition and $\mathrm{pH}$ shows complex behavior (Garidel et al., 2011). Hydrogen bond induced phase separation of anionic phospholipids has been observed previously (Redfern and Gericke, 2004; Dasgupta et al., 2009; Kooijman et al., 2009), and forms another intriguing example by which lipids may form important signaling platforms in biomembranes.

\section{THE PHOSPHOMONOESTER OF DGPP FOLLOWS THE ELECTROSTATIC-HYDROGEN BOND SWITCH MODEL}

Previously we showed for PA that the ionization behavior of its phosphomonoester headgroup sets it apart from other, more abundant, anionic lipids (Kooijman et al., 2005b, 2007). Upon initial ionization (to 1 negative charge) the remaining hydrogen in the headgroup is more stably bound by both covalent and electrostatic interactions (situation sketched in Figure 5 for the phosphomonoester of PA and DGPP). When a hydrogen bond is formed with this phosphomonoester headgroup this bonding will result in a competition for electrons and the last hydrogen in the headgroup is facilitated to deprotonate earlier compared to the situation lacking the hydrogen bond. This peculiar ionization behavior was summarized in the electrostatic-hydrogen bond switch model (Kooijman et al., 2007) and was instrumental in explaining the recent data for Opil of Loewen and coworkers (Young et al., 2010).

Here we show that like PA, the phosphomonoester headgroup of DGPP, follows this behavior exactly as might be expected (Kooijman and Burger, 2009; Kooijman and Testerink, 2010). Upon addition of PE to the membrane the $\mathrm{pKa}_{2}$ of DGPP's phosphomonoester is decreased significantly. This indicates that protein binding might be $\mathrm{pH}$ sensitive as was observed for some PA binding proteins. However no specific DGPP binding protein has been identified to date (aside from the observation that Opil has some affinity for DGPP; Young et al., 2010), but it will be important to check this possibility in the case genuine DGPP binding proteins are identified. $\mathrm{pH}$ sensitivity might point to significant cellular functions, and the linking of distinct signaling pathways as was observed for Opil binding to PA.

\section{THE $\mathrm{pKa}_{2}$ FOR DGPP IS LOWER THAN THAT OF PA}

We previously determined the $\mathrm{pKa}_{2}$ of $10 \mathrm{~mol} \%$ DOPA in a DOPC membrane and found it to be 7.9 (see Table 1). Here find that 5 mol\% DGPP has a $\mathrm{pKa}_{2}$ of 7.4 , significantly lower than that of 
PA. We confirmed that the $\mathrm{pKa}_{2}$ for $5 \mathrm{~mol} \%$ of DOPA in DOPC is around $\mathrm{pH} 7.9$ as well (data not shown) which indicates that this difference in $\mathrm{pKa}$ value is not due to a lower concentration of DGPP compared to DOPA. The most likely explanation of the difference in $\mathrm{pKa}_{2}$ between PA and DGPP is that the phosphomonoester of DGPP is located in a completely different region of the membrane. With the two phosphates stacked on top of each other, the phosphomonoester of DGPP penetrates much farther toward the aqueous phase than the phosphomonoester of PA and is likely to experience a much larger dielectric constant. A larger dielectric constant results in a lowering of the $\mathrm{pKa}$ (Cevc, 1990; Tocanne and Teissie, 1990).

The lower $\mathrm{pKa}_{2}$ for DGPP indicates that at constant $\mathrm{pH}$ DGPP carries more negative charge than the phosphomonoester of PA, ignoring for the moment the potential negative charge on the phosphodiester of DGPP (see discussion below). This higher charge would favor the interaction of cationic protein domains, and might result in a displacement of a PA bound protein to DGPP. However, DGPP is a membrane stabilizing phospholipid, and this displacement of the protein from PA to DGPP might thus (paradoxically) mean that the protein loses its affinity for the membrane thereby potentially turning of a PA signal. In this way both the charge and packing properties of DGPP might work in tandem to turn off a PA signal. Additionally, DGPP may have its own signaling properties, and through the highly negatively charged phosphomonoester is able to interact electrostatically (and via the electrostatic-hydrogen bond switch) with cationic proteins. It will thus be important to screen PA binding proteins for their propensity to bind DGPP.

\section{WHAT ABOUT THE CHARGE ON THE PHOSPHODIESTER OF DGPP?}

DGPP contains two phosphate groups (as pyrophosphate) in its headgroup which means that it can potentially carry three negative charges, compared to the two for PA. We also clearly observe the phosphodiester peak of DGPP and observe that its position does not change as significantly upon a change in $\mathrm{pH}$ as does the peak of the phosphomonoester (see Figure 4). This is not unexpected as the total shift induced by the deprotonation of the phosphodiester group likely only induces a modest shift in the chemical shift value. Consider for example the first $\mathrm{pKa}$ of the phosphomonoester which induces only a very modest shift in the chemical shift. When we compare the total shift in the chemical shift for the phosphomonoester $\left(\mathrm{pKa}_{1}\right)$ and the phosphodiester (over the entire $\mathrm{pH}$ range) the value is of the same magnitude. However when we plot the shift of the phosphodiester peak of DGPP as a function of $\mathrm{pH}$ for both titration curves we observe a behavior that identically follows the $\mathrm{pH}$ titration curve of the phosphomonoester. Figure 4 shows the shift of the phosphodiester of DGPP for the PC/PE matrix. In contrast the PE and PC peaks are essentially constant. Hence it is likely that the shift of the phosphodiester peak is induced solely by the decrease in shielding of the phosphomonoester group induced by deprotonation upon an increase in $\mathrm{pH}$. It is thus not possible to unambiguously define the ionization of the phosphodiester group based on our NMR data alone (Figures 3 and 4). From first principles it is highly likely however that it is still protonated until quite high $\mathrm{pH}$ values, and may even have a $\mathrm{pKa}$ well above the

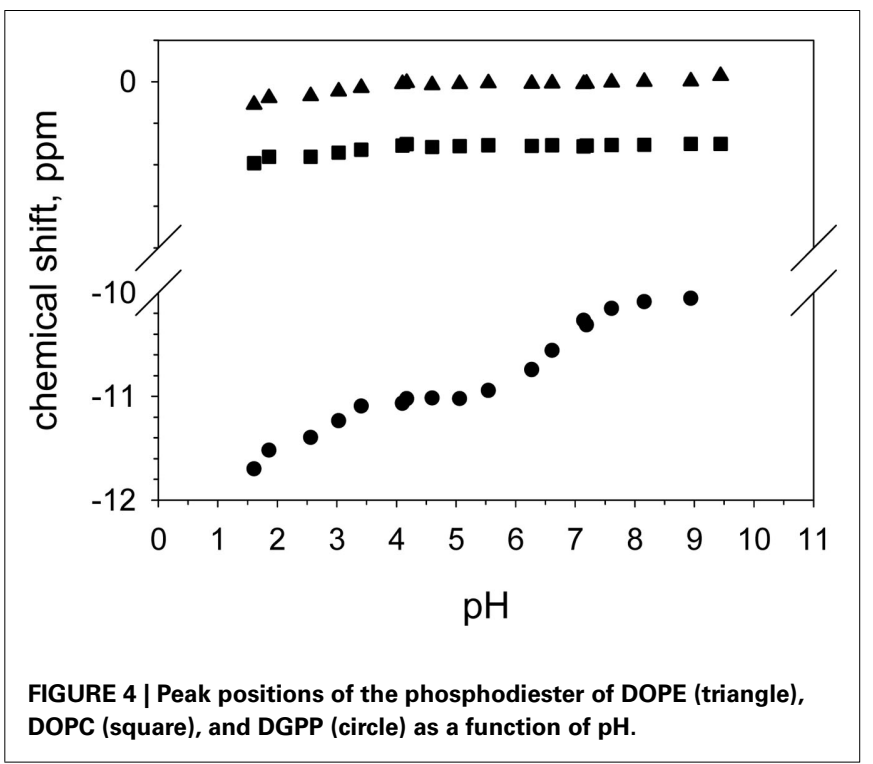

physiological range. This is understood from the fact that initial deprotonation of the headgroup will impede additional deprotonation since the negative charge will attract protons from the bulk to the membrane interface. These interfacial protons decrease the interfacial $\mathrm{pH}$ and hence decrease the likelihood that additional protons will deprotonate. Since the ionization behavior of the phosphodiester clearly indicates a $\mathrm{pKa}_{1}$ that is comparable to that of PA (see Table 1) it is not likely that the proton of both the phosphodiester and phosphomonoester are able to deprotonate at the same time. If the phosphodiester is protonated over the entire physiologically relevant $\mathrm{pH}$ range than DGPP will at most carry two negative charges in a biological membrane. This is contrary to the assumptions made by Villasuso and coworkers (Villasuso et al., 2010) who assumed that DGPP is able to carry three negative charges. Clearly though this point warrants further investigation; using optical spectroscopy we will investigate the ionization of the phosphodiester group in more detail.

\section{IMPLICATIONS FOR THE POTENTIAL ROLE OF DGPP IN BIOLOGICAL MEMBRANES}

The findings of our current study are schematically summarized in Figure 5. DGPP is formed via a phosphorylation of PA. Our data now shows that under physiological conditions this results in the conversion of an anionic lipid with negative membrane curvature to an anionic lipid with more negative charge and (essentially) zero membrane curvature. This conversion of PA to DGPP by PAK activity thus has significant implications for the original PA signal. PA binding proteins may no longer be able to bind to DGPP due to its lack of negative curvature, higher negative charge, and different position of the phosphomonoester in the headgroup interface of the lipid bilayer. Further work on the ionization state of the phosphodiester of DGPP and on the effect of additional charge and effective molecular shape effectors will likely provide additional insight into the physicochemical properties of the intriguing phospholipid DGPP. 

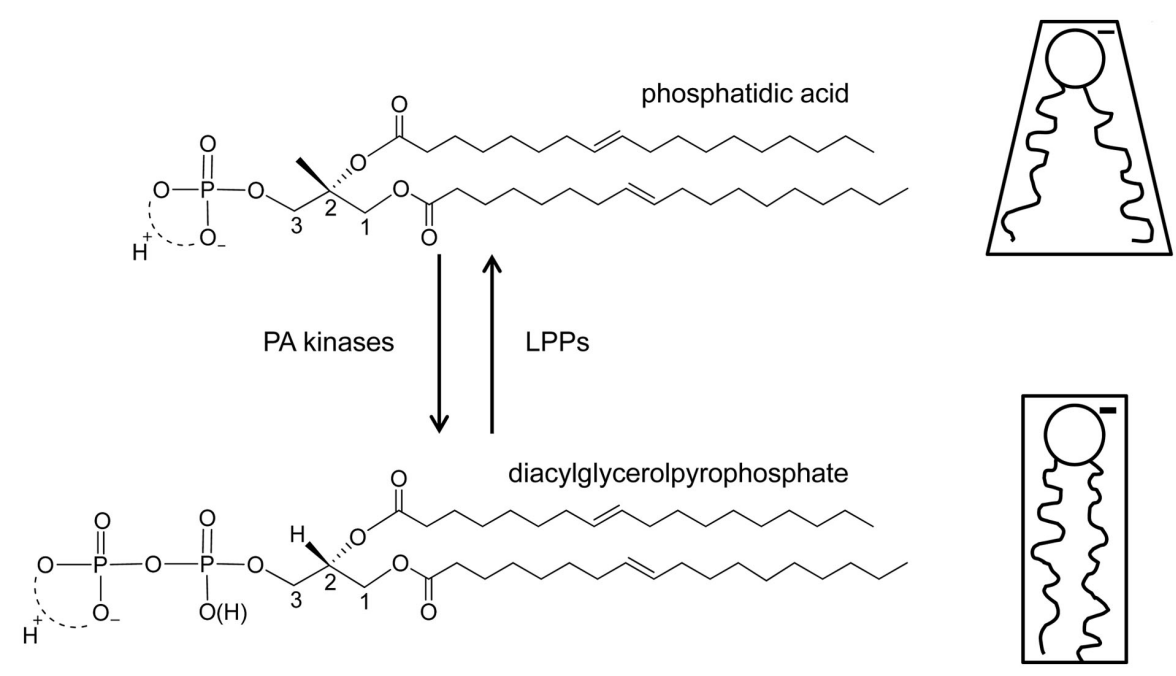

FIGURE 5 | Structure and functional model for DGPP. On the left the chemical structures of dioleoyl PA (top) and DGPP (bottom) are indicated. PA kinase activity is responsible for the synthesis of DGPP, but the enzyme responsible is currently unknown. Conversion of DGPP back to PA is facilitated by a family of known lipid phosphate phosphatases. On the right the effective molecular shape of PA and DGPP at physiological $\mathrm{pH}$ and salt $(\mathrm{NaCl})$ concentration is shown. The negative charge of both PA and DGPP is shown. In similar membrane environments (lipid composition) and under physiological conditions DGPP carries more negative charge then PA as indicated by the thicker "charge."

\section{MATERIALS AND METHODS MATERIALS}

1,2-Dioleoyl-sn-glycero-3-phosphatidylcholine (DOPC), 1-oleoyl2-hydroxy-sn-glycero-3-phosphocholine (LPC), 1,2-dioleoyl-snglycero-3-phosphatidyl-ethanolamine (DOPE), 1,2-dioleoyl-snglycero-3-phosphate (sodium salt; DOPA), 1,2-dielaidoyl-snglycero-3-phosphatidylethanolamine (DEPE), and 1,2-dioleoyldiacylglycerolpyrophosphate (DGPP) were purchased from Avanti Polar Lipids (Birmingham, AL, USA). All lipids were dissolved in chloroform, methanol (2:1 volumetric ratio) and were used as they were received from Avanti. Lipid purity of our stocks was regularly checked by HPTLC plates and judged to be better than 99\%, as one single spot on iodine stained plate. Water used in the experiments was of HPLC grade and purchased from Fisher Scientific. All buffer components were at least of HPLC grade.

\section{SAMPLE PREPARATION}

Differential scanning calorimetry samples were prepared by mixing appropriate amounts of lipid stock. The resulting lipid-solvent mixtures were then dried under a stream of $\mathrm{N}_{2}$ gas, at a temperature above the main melting transition for $\operatorname{DEPE}\left(T_{\mathrm{m}}=38^{\circ} \mathrm{C}\right)$. This is important as drying of lipid mixtures may result in demixing and thus two types of vesicle populations. Keeping the temperature of the solvent above the $T_{\mathrm{m}}$ of the lipids in the mixture should prevent this. These lipid films $(7 \mu \mathrm{mol}$ of total lipid) were then placed in a vacuum oven under high vacuum for at least $30 \mathrm{~min}$ at $45^{\circ} \mathrm{C}$ to remove residual traces of solvent. Lipid films were stored under $\mathrm{N}_{2}$ in a $-20^{\circ} \mathrm{C}$ freezer until used, usually within a week. Multilamellar dispersions were prepared by suspension of the lipid film in $1 \mathrm{~mL}$ of $150 \mathrm{mM}$ $\mathrm{NaCl}, 1 \mathrm{mM}$ EDTA, $20 \mathrm{mM}$ HEPES at pH 7.2 buffer solution. The sample was then heated in a water bath at a temperature above the main melting point $\left(T_{\mathrm{m}}\right)$ but below the $\mathrm{H}_{\mathrm{II}}$ phase transition temperature of DEPE, in this case approximately $45^{\circ} \mathrm{C}$, for $9 \mathrm{~min}$ and then vortexed for $1 \mathrm{~min}$. This was repeated two more times to make multilamellar vesicles. The sample and the reference buffer were then degassed before they were loaded in the calorimeter. Both the lipid stock solutions and the remainder of the sample used in the calorimeter were tested for concentration accuracy by phosphate assay (Rouser et al., 1970).

NMR samples, for $\mathrm{pH}$ titration purposes, were prepared by mixing appropriate amounts of lipid stock to prepare lipid films of the following lipid composition: A, $95 \mathrm{~mol} \%$ DOPC, and $5 \mathrm{~mol} \%$ DGPP; B, $47.5 \mathrm{~mol} \%$ DOPC, $47.5 \mathrm{~mol} \%$ DOPE, and $5 \mathrm{~mol} \%$ DGPP. The mixtures were dried to a thin lipid film on the bottom of specially made borosilicate glass tubes $(15 \mathrm{~mm}$ test tube size) in the rotary evaporator set-up at $45^{\circ} \mathrm{C}$. Lipid stocks and films were kept under an inert atmosphere of $\mathrm{N}_{2}$ gas during sample preparation. Hydrated lipid samples were prepared by suspending the lipid film in a buffer of the appropriate $\mathrm{pH}$. Buffers used were: $20 \mathrm{mM}$ Citric Acid, $30 \mathrm{mM}$ MES $4<\mathrm{pH}<6.7,50 \mathrm{mM}$ Tris $6.7<\mathrm{pH}<8.5,50 \mathrm{mM}$ CHES $8.5<\mathrm{pH}<10.5$, and contained $100 \mathrm{mM} \mathrm{NaCl}$ and $2 \mathrm{mM}$ EDTA to complex any traces of divalent cations. The hydrated lipid suspensions were flash frozen in dry ice cooled ethanol, and subsequently warmed to room temperature. The MLV suspension was occasionally vortexed as it thawed. This procedure assured full dispersion of the lipid film in the buffer. The $\mathrm{pH}$ of the lipid dispersion was measured with a Sentron intelli probe (RL Instruments, Manchaug, MA, USA) on a standard $\mathrm{pH}$ meter, which is particularly well-suited for samples with high lipid concentrations. This $\mathrm{pH}$, measured after lipid hydration, was used to construct the $\mathrm{pH}$ titration curves. The lipid dispersions were concentrated in a tabletop centrifuge $\left(14,900 \mathrm{rpm}, 1 \mathrm{~h} ; 20^{\circ} \mathrm{C}\right)$ and the (wet) lipid pellet was transferred to $4 \mathrm{~mm}$ zirconium MAS NMR sample tubes. 


\section{DIFFERENTIAL SCANNING CALORIMETRY}

Differential scanning calorimetry measurements of mixed multilamellar vesicles were carried out using a Microcal VP-DSC (Northampton, MA, USA). The scan rate was $0.75^{\circ} \mathrm{C} / \mathrm{min}$ and the total lipid concentration was $7 \mathrm{mM}$. This concentration was chosen in order to be able to clearly identify the change in enthalpy for the $\mathrm{L}_{\alpha}$ to $\mathrm{H}_{\text {II }}$ phase transition. The change in enthalpy for the $\mathrm{L}_{\alpha}$ to $\mathrm{H}_{\mathrm{II}}$ phase transition is much less energetic than the $\mathrm{L}_{\beta}-\mathrm{L}_{\alpha}$ phase transition, thus requiring a higher concentration. A total of six heating/cooling cycles (generally $20-80^{\circ} \mathrm{C}$, but $20-100^{\circ} \mathrm{C}$ for the LPC containing sample) were recorded and the third heating scan (fifth scan overall) was found to be representative.

\section{NMR SPECTROSCOPY}

NMR spectroscopy was performed as previously described (Kooijman et al., 2009). Briefly, MAS ${ }^{31} \mathrm{P}$ experiments were carried out using an observer pulse width of $5.25 \mu \mathrm{s}$. Static spectra to confirm phospholipid organization employed ${ }^{1} \mathrm{H}$ decoupling $\left(90^{\circ}, 22.25 \mu \mathrm{s}, 120 \mathrm{DB}\right)$. Spectra were recorded using a $1 \mathrm{~s}$ delay time between pulses. Samples were spun at the magic angle $\left(54.7^{\circ}\right)$ at $5 \mathrm{kHz}$ to average the CSA, and the chemical shift position of the lipids was recorded relative to an external $85 \% \mathrm{H}_{3} \mathrm{PO}_{4}$ standard. Under stable spinning conditions, typically, 25,000 scans were recorded. Experiments were carried out at a temperature of $25.0 \pm 1.0^{\circ} \mathrm{C}$. Static spectra, using low power proton decoupling (using the spinal-64 pulse program), were recorded in the same $4 \mathrm{~mm}$ CP MAS NMR probe, after the MAS spectra had been recorded. Typically between 50,000 and 100,000 scans were recorded.

\section{REFERENCES}

Appleton, T. G., Hall, J. R., Ralph, S. F., and Thompson, C. S. M. (1989). NMR study of acid-base equilibria and other reactions of ammineplatinum complexes with aqua and hydroxo ligands. Inorg. Chem. 28, 1989-1993.

Cevc, G. (1990). Membrane electrostatics. Biochim. Biophys. Acta 1031, 311-382.

Darwish, E., Testerink, C., Khalil, M., El-Shihy, O., and Munnik, T. (2009). Phospholipid signaling responses in salt-stressed rice leaves. Plant Cell Physiol. 50, 986-997.

Dasgupta, U., Bamba, T., Chiantia, S., Karim, P., Tayoun, A. N., Yonamine, I., Rawat, S. S., Rao, R. P., Nagashima, K., Fukusaki, E., Puri, V., Dolph, P. J., Schwille, P., Acharya, J. K., and Acharya, U. (2009). Ceramide kinase regulates phospholipase $\mathrm{C}$ and phosphatidylinositol 4, 5 , bisphosphate in phototransduction. Proc. Natl. Acad. Sci. U.S.A. 106, 20063-20068.

de Jong, C. F., Laxalt, A. M., Bargmann, B. O., de Wit, P. J., Joosten, M. H., and Munnik, T. (2004). Phosphatidic acid accumulation is an early response in the Cf-4/Avr4 interaction. Plant J. 39, 1-12. den Hartog, M., Musgrave, A., and induced phosphatidic acid and diacylglycerol pyrophosphate formation: a role for phospholipase $\mathrm{C}$ and $\mathrm{D}$ in root hair deformation. Plant J. 25, 55-65.

den Hartog, M., Verhoef, N., and Munnik, T. (2003). Nod factor and elicitors activate different phospholipid signaling pathways in suspension-cultured alfalfa cells. Plant Physiol. 132, 311-317.

Friebolin, H. (2011). Basic Oneand Two-Dimensional NMR Spectroscopy, 5th Edn. Weinheim: Wiley$\mathrm{VCH}$.

Fuller, N. L., and Rand, P. R. (2001). The influence of lysolipids on the spontaneous curvature and bending elasticity of phospholipid membranes. Biophys. J. 81, 243-254.

Garidel, P., Johann, C., and Blume, A. (2011). Non-ideal mixing and fluidfluid immiscibility in phosphatidic acid-phosphatidylethanolamine mixed bilayers. Eur. Biophys. J. 40, 891-905.

Gennis, R. B. (1989). Biomembrane, Molecular Structure and Function. New York: Spirnger-Verlag.

Hauser, H. (1989). Mechanism of spontaneous vesiculation. Munnik, T. (2001). Nod factor-

\section{DETERMINATION OF pKa VALUES}

The pKa value's for the phosphomonoester group of DGPP were determined using equation (1), which was derived from the Henderson-Hasselbalch equation using the assumption that the observed chemical shift values are weighted averages of the chemical shifts of the fully protonated, singly dissociated and doubly dissociated states (Appleton et al., 1989). The pKa values are determined utilizing a non-linear least squares fit procedure.

$\delta=\frac{\delta_{\mathrm{AB}}+\delta_{\mathrm{AA}} 10^{\left(\mathrm{pK \textrm {a } _ { 1 }}-\mathrm{pH}\right)}+\delta_{\mathrm{BB}} 10^{\left(\mathrm{pH}-\mathrm{pKa} \mathrm{a}_{2}\right)}}{1+10\left(\mathrm{pK \textrm {a } _ { 1 } - \mathrm { pH } )}+10^{\left(\mathrm{pH}-\mathrm{pKa} \mathrm{a}_{2}\right)}\right.}$

$\delta_{\mathrm{AA}}, \delta_{\mathrm{AB}}$, and $\delta_{\mathrm{BB}}$ are the chemical shifts of the fully protonated, single dissociated and double dissociated state respectively, $\delta$ is the measured $\mathrm{pH}$ dependent chemical shift and $\mathrm{pKa}_{1}$ and $\mathrm{pKa}_{2}$ are the first and second dissociation constant of the phosphomonoester.

\section{ACKNOWLEDGMENTS}

The authors are indebted to Mahinda Gangoda, and Zachery Graber for assistance with the NMR experiments. EEK acknowledges financial support from a Farris Family Fellowship, LR acknowledges the NSF REU program grant CHE-1004987. CT acknowledges support from the Netherlands Organization for Scientific Research (NWO; Vidi grant 700.56.429) and NGI Horizon project 93511011. Collaboration between the Kooijman and Testerink laboratories is supported by an ICAM (I2CAM) fellowship.

Proc. Natl. Acad. Sci. U.S.A. 86 5351-5355.

Jeannette, E., Paradis, S., and Zalejski, C. (2010). "Diacylglycerol pyrophosphate, a novel signaling lipid," in Lipid Signaling in Plants, ed. T. Munnik (Berlin: Springer-Verag), 263-276.

Katagiri, T., Ishiyama, K., Kato, T., Tabata, S., Kobayashi, M., and Shinozaki, K. (2005). An important role of phosphatidic acid in ABA signaling during germination in Arabidopsis thaliana. Plant J. 43, 107-117.

Kooijman, E. E., and Burger, K. N. (2009). Biophysics and function of phosphatidic acid: a molecular perspective. Biochim. Biophys. Acto 1791, 881-888.

Kooijman, E. E., Chupin, V., de Kruijff, B., and Burger, K. N. (2003). Modulation of membrane curvature by phosphatidic acid and lysophosphatidic acid. Traffic 4, 162-174.

Kooijman, E. E., Chupin, V., Fuller, N. L. Kozlov, M. M., de Kruijff, B., Burger, K. N., and Rand, P. R. (2005a). Spontaneous curvature of phosphatidic acid and lysophosphatidic acid. Biochemistry 44, 2097-2102.

Kooijman, E. E., Carter, K. M., van Laar, E. G., Chupin, V., Burger, K.
N., and de Kruijff, B. (2005b). What makes the bioactive lipids phosphatidic acid and lysophosphatidic acid so special? Biochemistry 44, 17007-17015

Kooijman, E. E., King, K. E., Gangoda, M., and Gericke, A. (2009). Ionization properties of phosphatidylinositol polyphosphates in mixed model membranes. Biochemistry 48, 9360-9371.

Kooijman, E. E., Sot, J., Montes, L. R., Alonso, A., Gericke, A., de Kruijff, B., Kumar, S., and Goni, F. M. (2008). Membrane organization and ionization behavior of the minor but crucial lipid ceramide1-phosphate. Biophys. J. 94, 4320-4330.

Kooijman, E. E., and Testerink, C. (2010). "Phosphatidic acid: an electrostatic/hydrogen-bond switch?" in Lipid Signaling in Plants, ed. T. Munnik (Berlin: Springer Verlag), 203-222.

Kooijman, E. E., Tieleman, D. P., Testerink, C., Munnik, T., Rijkers, D. T., Burger, K. N., and de Kruijff, B. (2007). An electrostatic/hydrogen bond switch as the basis for the specific interaction of phosphatidic acid with proteins. J. Biol. Chem. 282, 11356-11364. 
Ktistakis, N. T. (2010). Lipid signaling and homeostasis: PA- is better than PA-H, but what about those PIPs? Sci. Signal. 3, pe46.

Levental, I., Cebers, A., and Janmey, P. A. (2008a). Combined electrostatics and hydrogen bonding determine intermolecular interactions between polyphosphoinositides. J. Am. Chem. Soc. 130, 9025-9030.

Levental, I., Janmey, P. A., and Cebers, A. (2008b). Electrostatic contribution to the surface pressure of charged monolayers containing polyphosphoinositides. Biophys. J. 95, 1199-1205.

Marsh, D. (2012). Hanbook of Lipid Bilayers. Second edition, Boca Raton, FL: CRC Press.

Munnik, T., Arisz, S. A., De Vrije, T., and Musgrave, A. (1995). G protein activation stimulates phospholipase D signaling in plants. Plant Cell 7, 2197-2210.

Munnik, T., de Vrije, T., Irvine, R. F., and Musgrave, A. (1996). Identification of diacylglycerol pyrophosphate as a novel metabolic product of phosphatidic acid during G-protein activation in plants. J. Biol. Chem. 271, 15708-15715.

Munnik, T., Meijer, H. J., Ter Riet, B., Hirt, H., Frank, W., Bartels, D., and Musgrave, A. (2000). Hyperosmotic stress stimulates phospholipase $\mathrm{D}$ activity and elevates the levels of phosphatidic acid and diacylglycerol pyrophosphate. Plant J. 22, 147-154.

Orij, R., Brul, S., and Smits, G. J. (2011). Intracellular $\mathrm{pH}$ is a tightly controlled signal in yeast. Biochim. Biophys. Acta 1810, 933-944.

Paradis, S., Villasuso, A. L., Aguayo, S. S., Maldiney, R., Habricot, Y., Zalejski, C., Machado, E., Sotta, B., Miginiac, E., and Jeannette, E. (2011). Arabidopsis thaliana lipid phosphate phosphatase 2 is involved in abscisic acid signalling in leaves. Plant Physiol. Biochem. 49, 357-362.

Pical, C., Westergren, T., Dove, S. K., Larsson, C., and Sommarin, M. (1999). Salinity and hyperosmotic stress induce rapid increases in phosphatidylinositol 4,5-bisphosphate, diacylglycerol pyrophosphate, and phosphatidylcholine in Arabidopsis thaliana cells. J. Biol. Chem. 274, 38232-38240.

Pierrugues, O., Brutesco, C., Oshiro, J., Gouy, M., Deveaux, Y., Carman, G. M., Thuriaux, P., and Kazmaier, M. (2001). Lipid phosphate phosphatases in Arabidopsis. Regulation of the AtLPP1 gene in response to stress. J. Biol. Chem. 276, 20300-20308.

Rand, R. P., Fuller, N. L., Gruner, S. M., and Parsegian, V. A. (1990). Membrane curvature, lipid segregation, and structural transitions for phospholipids under dual-solvent stress. Biochemistry 29, 76-87.

Redfern, D. A., and Gericke, A. (2004). Domain formation in phosphatidylinositol monophosphate/phosphatidylcholine mixed vesicles. Biophys. J. 86, 2980-2992.

Roth, M. G. (2008). Molecular mechanisms of PLD function in membrane traffic. Traffic 9, 1233-1239.

Rouser, G., Fkeischer, S., and Yamamoto, A. (1970). Two dimensional then layer chromatographic separation of polar lipids and determination of phospholipids by phosphorus analysis of spots. Lipids 5, 494-496.

Shin, J. J., and Loewen, C. J. (2011). Putting the $\mathrm{pH}$ into phosphatidic acid signaling. BMC Biol. 9, 85 . doi:10.1186/1741-7007-9-85

Stace, C. L., and Ktistakis, N. T. (2006). Phosphatidic acid- and phosphatidylserine-binding proteins. Biochim. Biophys. Acta 1761,913-926.
Swairjo, M. A., Seaton, B. A., and Roberts, M. F. (1994). Effect of vesicle composition and curvature on the dissociation of phosphatidic acid in small unilamellar vesicles - a 31PNMR study. Biochim. Biophys. Acta 1191, 354-361.

Szule, J. A., Fuller, N. L., and Rand, R. P. (2002). The effects of acyl chain length and saturation of diacylglycerols and phosphatidylcholines on membrane monolayer curvature. Biophys. J. 83, 977-984.

Testerink, C., and Munnik, T. (2011). Molecular, cellular, and physiological responses to phosphatidic acid formation in plants. J. Exp. Bot. 62, 2349-2361.

Tocanne, J. F., and Teissie, J. (1990). Ionization of phospholipids and phospholipid-supported interfacial lateral diffusion of protons in membrane model systems. Biochim. Biophys. Acta 1031, 111-142.

van der Luit, A. H., Piatti, T., van Doorn, A., Musgrave, A., Felix, G., Boller, T., and Munnik, T. (2000). Elicitation of suspension-cultured tomato cells triggers the formation of phosphatidic acid and diacylglycerol pyrophosphate. Plant Physiol. 123, 1507-1516.

Villasuso, A. L., Wilke, N., Maggio, B. and Machado, E. (2010). The surface organization of diacylglycerol pyrophosphate and its interaction with phosphatidic acid at the airwater interface. Chem. Phys. Lipids 163, 771-777.

Young, B. P., Shin, J. J., Orij, R., Chao, J. T., Li, S. C., Guan, X. L., Khong, A., Jan, E., Wenk, M. R., Prinz, W. A., Smits, G. J., and Loewen, C. J. (2010). Phosphatidic acid is a pH biosensor that links membrane biogenesis to metabolism. Science 329, 1085-1088.
Zalejski, C., Paradis, S., Maldiney, R., Habricot, Y., Miginiac, E., Rona, J. P., and Jeannette, E. (2006). Induction of abscisic acid-regulated gene expression by diacylglycerol pyrophosphate involves $\mathrm{Ca} 2+$ and anion currents in Arabidopsis suspension cells. Plant Physiol. 141, 1555-1562.

Zalejski, C., Zhang, Z., Quettier, A. L., Maldiney, R., Bonnet, M., Brault, M. Demandre, C., Miginiac, E., Rona, J. P., Sotta, B., and Jeannette, E. (2005). Diacylglycerol pyrophosphate is a second messenger of abscisic acid signaling in Arabidopsis thaliana suspension cells. Plant J. 42, 145-152.

Conflict of Interest Statement: The authors declare that the research was conducted in the absence of any commercial or financial relationships that could be construed as a potential conflict of interest.

Received: 11 January 2012; paper pending published: 31 January 2012; accepted: 18 February 2012; published online: 20 March 2012.

Citation: Strawn L, Babb A, Testerink C and Kooijman EE (2012) The physical chemistry of the enigmatic phospholipid diacylglycerol pyrophosphate. Front. Plant Sci. 3:40. doi: 10.3389/fpls.2012.00040

This article was submitted to Frontiers in Plant Physiology, a specialty of Frontiers in Plant Science.

Copyright (C) 2012 Strawn, Babb, Testerink and Kooijman. This is an openaccess article distributed under the terms of the Creative Commons Attribution Non Commercial License, which permits non-commercial use, distribution, and reproduction in other forums, provided the original authors and source are credited. 